\title{
Diagnosis and Management of Autonomic Failure in Neurodegenerative Disorders
}

\author{
Luisa Sambati ${ }^{a}$ b Giovanna Calandra-Buonaura ${ }^{a}$ b Andrea Doria ${ }^{b}$ Pietro Cortelli ${ }^{a}$ b \\ ${ }^{a}$ IRCCS Institute of Neurological Sciences of Bologna and ${ }^{b}$ Department of Biomedical and Neuromotor Sciences, \\ University of Bologna, Italy
}

\section{Key Words}

Neurodegenerative disease $\cdot$ Autonomic nervous system .

Cardiovascular reflex tests

\begin{abstract}
Background:One of the hallmarks of thea-synucleinopathies is the degeneration of the autonomic nervous system. Summary: This review discusses the diagnosis and management of cardiovascular autonomic failure within the context of the a-synucleinopathies. In addition, it outlines the utility of various laboratory assessments including cardiovascular reflex tests for the differential diagnoses of these disorders, as well as general disease management strategies. Key Messages: Laboratory investigations assessing the autonomic control of the cardiovascular system are useful in the differential diagnosis of a-synucleinopathies, especially in early stages of disease. Clinical Implications: The characterization of the different features of AF in patients with a-synucleinopathies is challenging because it might help to improve the accuracy of the differential diagnosis between these diseases at onset. Further cardiovascular AF has been demonstrated to have a negative prognostic role in a-synucleinopathies, therefore an early detection of cardiovascular dysautonomia allows to positively impact the disease course guiding the appropriate therapy.

(c) 2014 S. Karger AG, Basel
\end{abstract}

(c) 2014 S. Karger AG, Base

0014-3022/14/0732-0126\$39.50/0

\section{Introduction}

Progressive central nervous system (CNS) degeneration due to the aggregation and deposition of misfolded proteins characterizes neurodegenerative disorders [1]. Under these conditions, the clinical features are related to the progression of the pathological processes in different areas of the CNS, and some components of the autonomic nervous system (ANS) can be involved. The latter is that part of the nervous system that regulates visceral functions and integrated processes that control vital functions, encompassing any nervous activity that does not reach the level of consciousness [2].

The ANS is composed of the Central Autonomic Network, including several interconnected areas of the CNS that receive and integrate humoral, viscerosensory, and environmental inputs to generate specific autonomic, endocrine, and somatomotor responses, and of an efferent pathway, which comprises the sympathetic and parasympathetic nervous systems. Each efferent pathway is composed of a two-neuron system consisting of pre- and postganglionic neurons, which make synapses with the target organ [3].

The ANS is typically involved in $\alpha$-synucleinopathies, degenerative disorders characterized by the presence of intracellular inclusions containing a-synuclein, which also affect the CNS (multiple system atrophy [MSA], Par- 


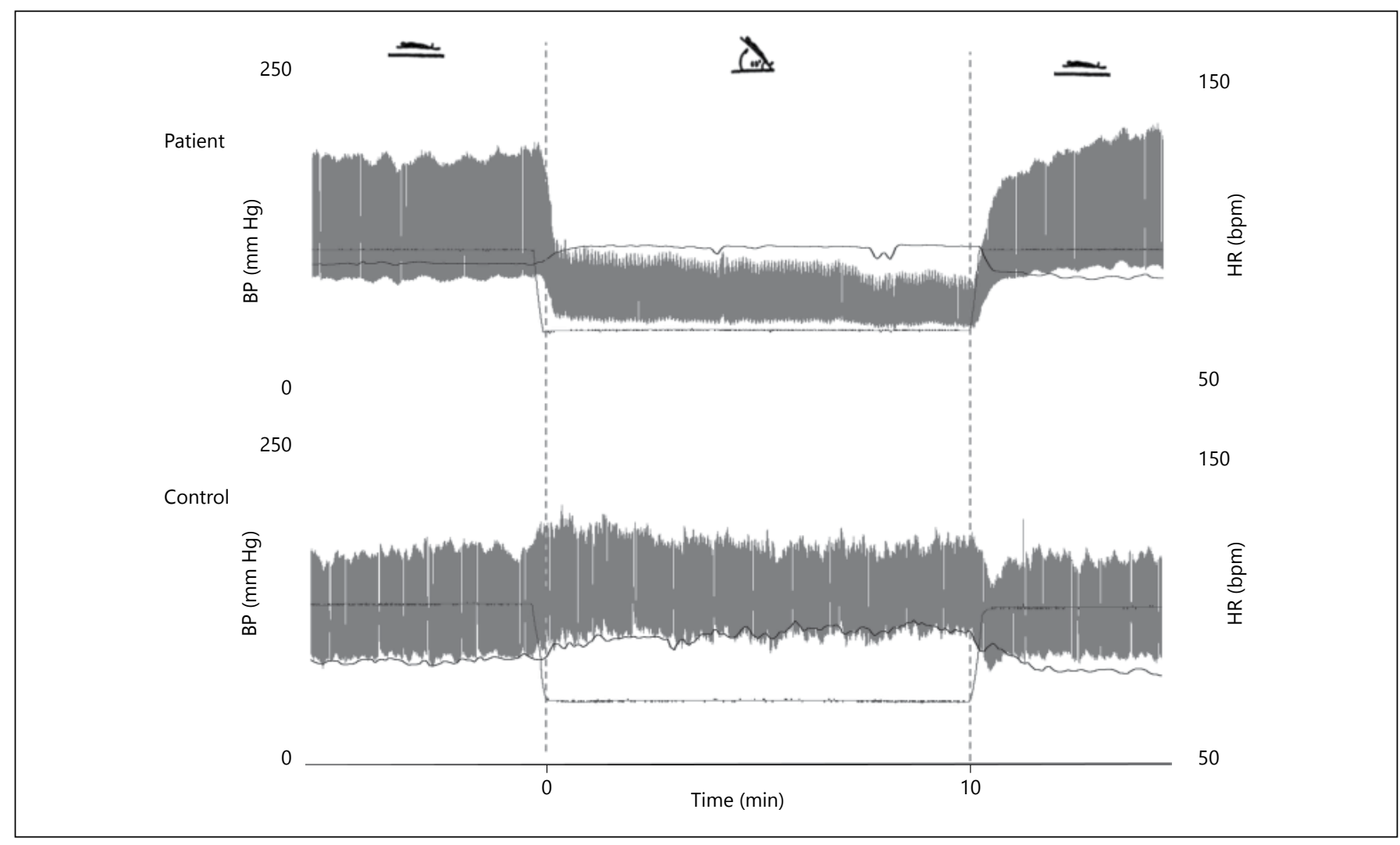

Fig. 1. Cardiovascular responses to standing during head up tilt test in a patient presenting with Parkinson's disease and orthostatic hypotension and in a normal control. $\mathrm{BP}=$ Blood pressure; $\mathrm{HR}=$ heart rate; $\mathrm{bpm}=$ beats per minute; $\min =$ minutes.

kinson's disease $[\mathrm{PD}]$, dementia with Lewy bodies [LBD]), or only the peripheral nervous system (pure autonomic failure [PAF]) [4].

In degenerative disorders, the ANS could be entirely or selectively (either the sympathetic or the parasympathetic component) affected. The autonomic involvement in a-synucleinopathies mainly results in a failure of the sympathetic branch of ANS and only affects the postganglionic component in PAF, mainly the postganglionic component in $\mathrm{LBD}$ and $\mathrm{PD}$, and the preganglionic one in MSA.

Sympathetic autonomic failure (AF) is clinically characterized by cardiovascular AF, sexual dysfunction, and neurogenic bladder and bowel. From a clinical perspective, AF is mandatory for a diagnosis of PAF and MSA [5], while symptoms of AF are reported as clinical supportive features of LBD [6,7]. As cardiovascular AF has been demonstrated to negatively impact the disease course in a-synucleinopathies [8], in this review we will discuss current knowledge on clinical and instrumental diagnosis, and management of cardiovascular AF that presents in the context of these diseases. In addition, we will focus on the utility of the laboratory investigations for assessing the autonomic control of the cardiovascular system (cardiovascular reflex tests) in the differential diagnosis of these disorders, especially in early disease stages.

\section{Clinical Observation}

The cardinal sign of sympathetic neurocirculatory failure is orthostatic hypotension $(\mathrm{OH})$, which is defined as a sustained reduction in systolic blood pressure (SBP) of at least $20 \mathrm{~mm} \mathrm{Hg}$ or in diastolic BP (DBP) of at least 10 $\mathrm{mm} \mathrm{Hg}$, within three minutes of standing up or head-up tilt to at least $60^{\circ}$ on a tilt table (fig. 1) [9]. OH may be asymptomatic or symptomatic. Clinical manifestations of $\mathrm{OH}$ are reported in table 1.

In MSA, OH is almost invariably present during the disease course; however, less than $50 \%$ of patients experience syncope. $\mathrm{OH}$ may occur early in the course of the disease, and in these cases is associated with a shorter survival time $[10,11]$. Similarly, recent studies demonstrated 
Table 1. Signs and symptoms of orthostatic hypotension

Dizziness, weakness, lethargy, fatigue

Visual disturbances: blurred vision, tunnel scotoma, greying out, blacking out

Paracervical and suboccipital ('coat hanger') ache, lower back/buttock ache

Falls and syncope

Oliguria

that in long-surviving patients, cardiovascular dysautonomia occurred late in the disease course suggesting that late onset of cardiovascular AF in MSA patients may be a favorable prognostic factor $[12,13]$.

In the diagnosis of PD [6], early and severe autonomic involvement is considered an exclusion criterion. However, it has been observed that $\mathrm{OH}$ can occur early in the disease course, even before the hallmark motor phase of $\mathrm{PD}$, and autopsy-proven PD patients misdiagnosed as having PAF or MSA in life have been described $[14,15]$. Goldstein reported that, in a cohort of 35 PD patients with $\mathrm{OH}, 60 \%$ had documentation of $\mathrm{OH}$ as an early finding (at least one year after the onset of motor symptoms) [14]. Thus, $\mathrm{OH}$ has been proposed as a non-motor feature in PD [7], although its prevalence and severity are still controversial. A recent meta-analysis of 25 studies reported a $30 \%$ prevalence of $\mathrm{OH}$ at any stage of disease in $\mathrm{PD}$ with a large statistical heterogeneity between studies. Several individual studies reported in the meta-analysis have shown that $\mathrm{OH}$ occurs in older patients and at a later stage of the disease, but this information could not be analyzed because data from individual patients were not reported. Dopaminergic treatment can increase $\mathrm{OH}$ risk in $\mathrm{PD}$, but it may occur even in the absence of treatment [16].

In LBD, AF is considered a supportive diagnostic feature. In a series of 29 cases with pathological confirmation, the frequency of $\mathrm{OH}$ (defined as SBP lower than 100 $\mathrm{mm} \mathrm{Hg}$ when standing) including cases without episodes of syncope reached $66 \%$, while syncopal episodes occurred in $28 \%$ of patients [17]. Thaisetthawatkul and coauthors compared autonomic dysfunctions in LBD patients with MSA and PD patients. The severity of AF was evaluated through the rating of the frequency and scoring of severity of $\mathrm{OH}$ and through the scoring of the Composite Autonomic Severity Score (CASS) [18]. They found that the severity of AF in LBD patients was intermediate between MSA and PD patients, with MSA patients being the most severely affected $[17,19,20]$.

PAF is a rare, idiopathic sporadic disorder characterized by severe $\mathrm{OH}$ and frequent episodes of syncope, a relatively slow and unchanging heart rate (HR), reduced sweating, erectile dysfunction, and constipation, without symptoms or signs of central neurodegeneration.

Interestingly, patients presenting with $\mathrm{PAF}$, after many years of 'pure' autonomic involvement may develop signs of CNS dysfunction (Parkinsonism and/or dementia) suggesting a common pathogenesis between $\mathrm{PAF}, \mathrm{PD}$ and LBD [21].

In about $50 \%$ of patients with $\mathrm{OH}$, supine hypertension (SH), defined as a SBP over $150 \mathrm{~mm} \mathrm{Hg}$ or DBP over $90 \mathrm{~mm} \mathrm{Hg}$, has been reported [7] and similarly seems to be related to the impairment of cardiovascular autonomic control. The association between $\mathrm{OH}$ and $\mathrm{SH}$ defines the 'Orthostatic Hypotension-Supine Hypertension' syndrome. $\mathrm{SH}$ can occur at any time during the day when the patient is lying down or even when semi-recumbent, but it is especially prevalent at night during sleep. Since drugs used to reverse $\mathrm{OH}$ can worsen $\mathrm{SH}$ [22] and nocturnal SH is linked to an increased cardiovascular risk and may contribute to ventricular hypertrophy, renal dysfunction and intracerebral hemorrhage, it is crucial to recognize $\mathrm{SH}$ and treat it accordingly.

\section{Laboratory Assessment}

Dysfunction of the autonomic control of the cardiovascular system can be evaluated through noninvasive physiological, biochemical, and pharmacological tests (table 2). These tests should be performed when $\mathrm{OH}$ is suspected on the basis of the medical history and the neurological examination (fig. 2). These tests simulate daily activities that induce stress conditions on the autonomic control of the cardiovascular system.

Physiological tests evaluate the integrity of both the effector branches, or selectively of either the sympathetic or the parasympathetic one. The head-up tilt test (HUT) and the valsalva maneuver (VM) assess the functioning of both branches. The heart rate response to deep breathing allows the evaluation of the cardiovagal efferent system and its relationships with breathing. Sustained isometric muscle contraction (sustaining a fixed, isometric handgrip contraction for $3 \mathrm{~min}$ at $30 \%$ of maximum effort), cold water immersion (immersing a hand in a container of icy water for 1-3 min), and mental stress tests (performing mental arithmetic, e.g., subtracting 7 serially from 100 and the Stroop color word-naming test) are pressure stimuli and markers of the vascular efferent sympathetic system function [23]. 
Table 2. Laboratory assessments for evaluating dysfunctional autonomic control of the cardiovascular system

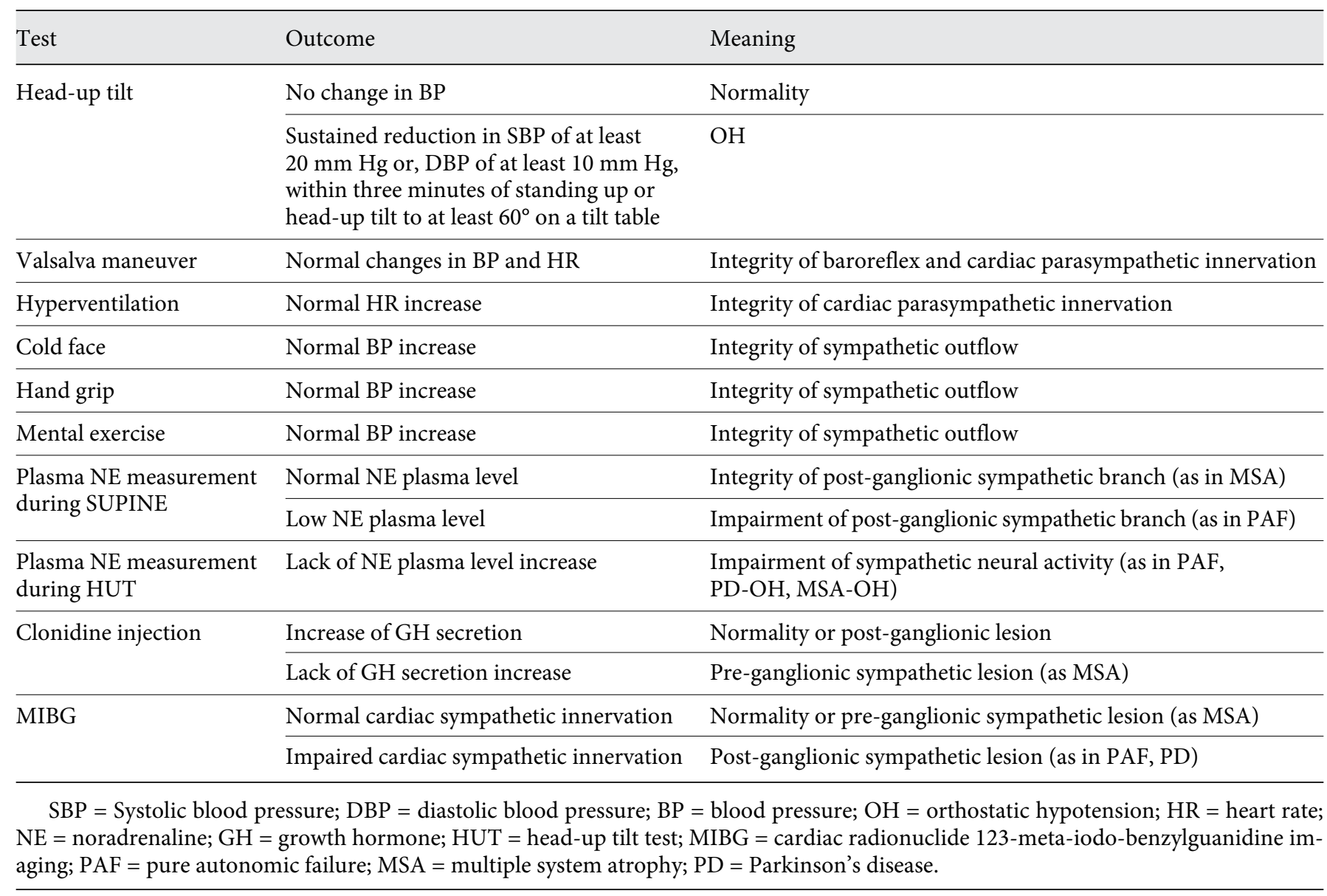

If the medical history is suggestive of $\mathrm{OH}$, cardiovascular responses to standing should be investigated first by the active standing test and, if this is negative, the passive HUT test is recommended [24].

$\mathrm{VM}$ is performed by blowing through a mouthpiece connected to a mercury manometer at $40 \mathrm{~mm} \mathrm{Hg}$ for $15 \mathrm{~s}$. VM consists of four phases. Phase I starts at the beginning of the respiratory strain and is consistent with a transient increase in arterial BP (ABP). Phase IIa is characterized by a fall in ABP due to impaired atrial filling of the heart. The successive phase IIb is triggered by the sympathetic reflex activation due to the fall in $\mathrm{ABP}$ and leads to a rise in $\mathrm{ABP}$ and an increase in heart rate. At the end of the strain, the sudden decrease in intra-thoracic pressure leads to phase III, where a transient decrease in ABP occurs. This is followed by phase IV, characterized by an overshoot of $\mathrm{ABP}$ above baseline, due to the recovery of a normal cardiac output against a still elevated sympathetic tone resulting in an increased total peripheral resis- tance. A transient bradycardia is the normal response to the ABP overshoot of phase IV (fig. 3) [25].

In patients with chronic primary AF, a particular pattern of pressure responses to the VM has been reported $[7,26]$. In these patients, ABP decreases progressively during phase II and does not exceed the baseline value during phase IV. This pattern indicates a sympathetic cardiovascular failure in response to decreased cardiac filling [7].

Recent studies [15, 27-29] have reported that the severity and distribution of the autonomic dysfunction may differentiate PD from MSA. Some of these studies used CASS as a quantitative index measure of overall autonomic dysfunction on standard autonomic testing, including the cardiovascular reflex test. Kimpinsky reported that CASS total scores showed good sensitivity (89\%) but a moderate specificity (70\%) in discriminating MSA from PD [27]. Lipp et al. reported that autonomic indices (CASS and the thermoregulatory sweat test, TST) were 


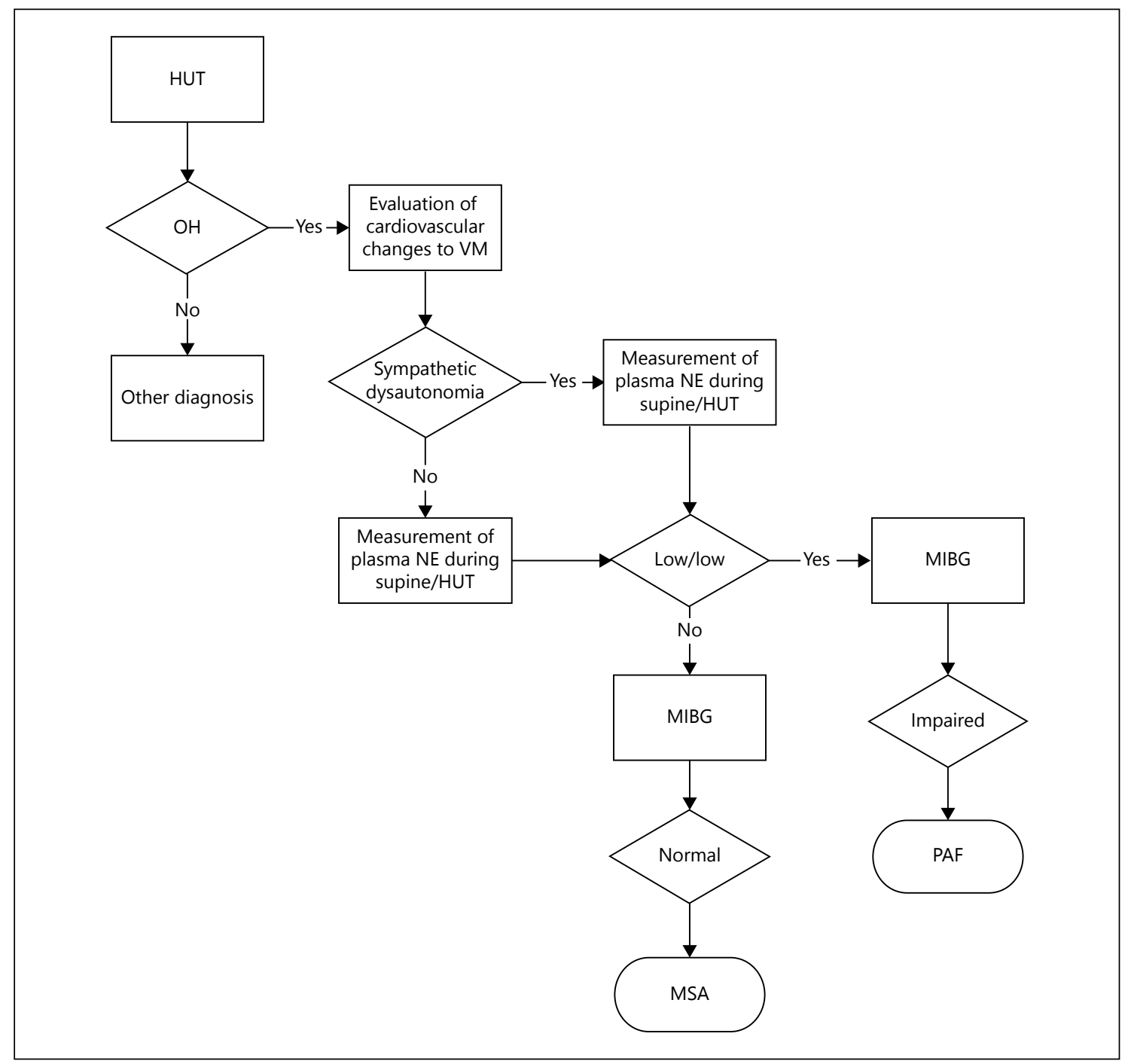

Fig. 2. Laboratory assessment in the differential diagnosis between PAF and MSA. HUT = Head-up tilt test; VM = valsalva maneuver; $\mathrm{OH}=$ orthostatic hypotension; $\mathrm{NE}=$ norepinephrine; $\mathrm{MIBG}=$ cardiac radionuclide 123-meta-iodo-benzylguanidine imaging; PAF = pure autonomic failure; MSA = multiple system atrophy.

Fig. 3. Valsalva maneuver in a patient with Parkinson's disease (a) and orthostatic hypotension, compared to a normal control (b). $\mathrm{BP}=$ Blood pressure; $\mathrm{HR}=$ heart rate; bpm = beats per minute; $\mathrm{EP}=$ expiration period; $\mathrm{sec}=$ seconds; $\min =$ minutes.

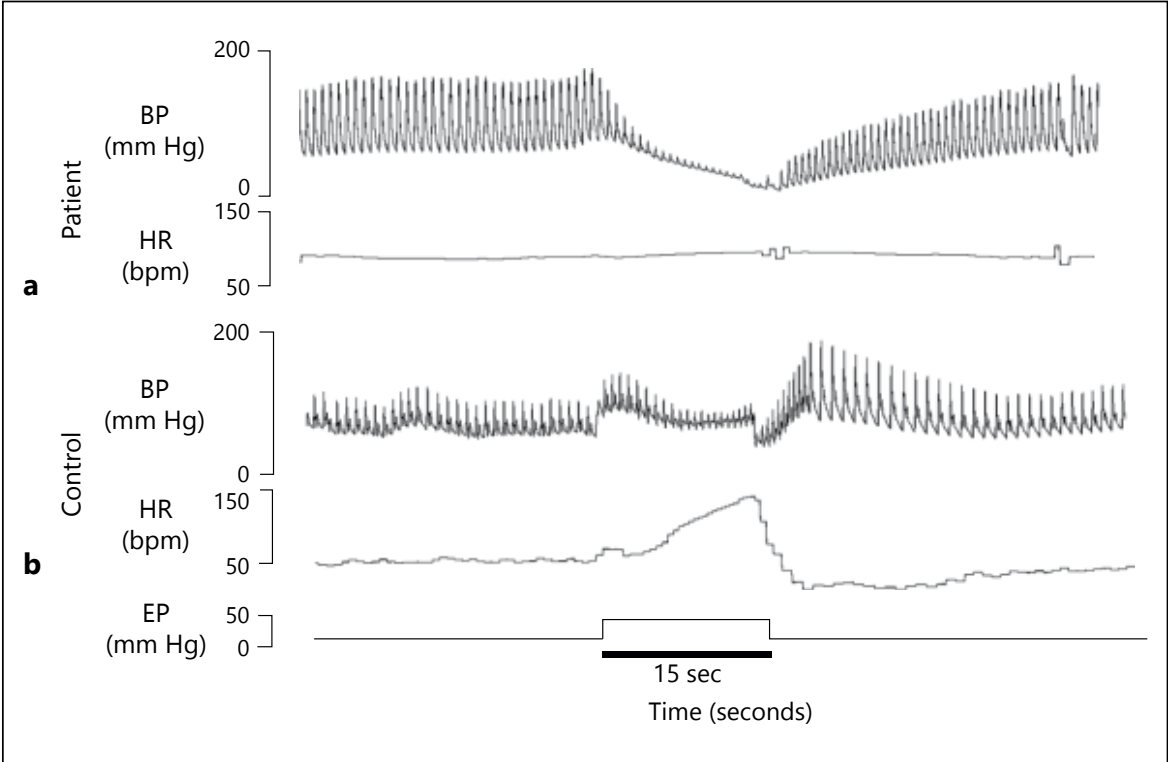


significantly more abnormal in MSA than in PD at onset, and that these differences were sustained and became greater at a further evaluation after 12 months. Furthermore, the percentage of sweating at the TST can distinguish between the two diseases [15] but with a minimal overlap between the scores of the different patients.

Unfortunately, these studies were performed on small samples of patients, mainly in advanced stages of the disease, when the maneuvers are problematic to perform, and in patients on medication. Furthermore, none of these studies had pathological confirmation of the disease.

As cardiovascular reflex tests are noninvasive, inexpensive, and reproducible, their accuracy for the differential diagnosis of $\alpha$-synucleinopathies should be confirmed in a larger prospective autopsy-confirmed study.

The evaluation of epinephrine and norepinephrine (NE) plasma levels provides the measure of adrenal medullary activity and sympathetic neural activity, respectively. Measurement of plasma NE is useful to differentiate PAF from MSA. In PAF, the supine basal concentration of plasma NE is lower compared to MSA in which supine values are within the normal range. In both, however, there is an attenuation or lack of physiological rise in plasma NE during HUT.

$\mathrm{PD}$ patients with $\mathrm{OH}$ have lower NE supine concentrations than those without $\mathrm{OH}$. Nevertheless, PD patients with $\mathrm{OH}$ do not have significantly lower NE supine concentrations compared to healthy people of similar age, and the concentrations are higher than those in patients with PAF $[2,7]$. NE concentrations are similar in PD patients with $\mathrm{OH}$ and in MSA patients. During HUT, PAF patients, PD patients with $\mathrm{OH}$, and MSA patients with OH have sharp orthostatic increments in plasma NE concentrations, while $\mathrm{PD}$ patients without $\mathrm{OH}$ have an increase of $60 \%$ or more. In LBD, the plasma NE concentration compared to PD patients is lower at rest and the response of plasma NE to HUT is blunted.

Pharmacological tests can determine the degree of sensitivity of different receptors and the functional integrity of ANS components. A pharmacological challenge with the $\alpha$-2 adrenoreceptor agonist clonidine causes the stimulation of growth hormone release. It is useful for differentiating PAF patients, who exhibit a rise in growth hormone serum concentrations after clonidine injection, from MSA patients, in whom these concentrations remain stable [2].

Cardiac radionuclide 123-meta-iodo-benzylguanidine (MIBG) imaging assesses cardiac sympathetic innervations. The distribution of cardiac sympathetic nerves and the uptake function at the nerve ending is quantified with the heart to mediastinum $(\mathrm{H} / \mathrm{M})$ ratio. MIBG uptake is generally impaired in PAF, LBD, and PD patients with $\mathrm{OH}$ and in about one half of PD patients without $\mathrm{OH}$. These abnormalities are not usually present in MSA patients, but impaired myocardial innervations in these patients have been reported [27, 30].

The sensitivity and specificity of MIBG scintigraphy are high (near 90\%) with respect to highlighting cardiac denervation. A recent meta-analysis showed that the pooled sensitivity to differentiate PD from other neurodegenerative parkinsonisms by a delayed $\mathrm{H} / \mathrm{M}$ ratio is $89.7 \%$ and the specificity is $82.6 \%$ [31]. Treglia and colleagues found a pooled sensitivity and specificity of $89 \%$ (95\% CI: $86-91 \%)$ and $77 \%$ (95\% CI: 68-84\%), respectively, in differential diagnosis between PD and MSA [30]. In another recent study, pooled sensitivity and specificity for differentiating PD and MSA were 90.2\% (95\% CI: 84.4\%, 93.9\%) and $81.9 \%$ (95\% CI: 56.1\%, 94.1\%), respectively [32].

Several studies have used 6-[18F] fluorodopamine positron emission tomography to evaluate the noradrenergic denervation in cardiac and extracardiac organs. In patients with $\mathrm{PAF}, \mathrm{PD}$, and $\mathrm{PD}$ with $\mathrm{OH}$, loss of sympathetic innervations was detected in the heart, in the thyroid gland and in the renal cortex.

Finally, as $\mathrm{OH}$ in these patients is often linked to $\mathrm{SH}$, 24-h ambulatory BP monitoring may be useful for evaluating $\mathrm{SH}$, especially during the nighttime [22].

\section{Prevention and Management}

Unfortunately, there are no targeted treatments for modifying the course of any of these disorders [33]. At present, treatment should be directed toward ameliorating the disease symptoms and resolving $\mathrm{OH}$ symptoms in patients with asymptomatic $\mathrm{OH}$, thus improving the patient's functional status and reducing the risk of falls due to syncope. To achieve a balance between $\mathrm{OH}$ and $\mathrm{SH}$, a goal of SBP of $100 \mathrm{~mm} \mathrm{Hg}$ while standing is recommended, at least in the morning [7].

In patients with asymptomatic $\mathrm{OH}$, a stepwise treatment starting with non-pharmacological measures (table 3) could prevent the need for pharmacological treatment. Non-pharmacological treatment measures are also recommended for patients with symptomatic $\mathrm{OH}$.

Pharmacological treatment is required when lifestyle measures are not effective, in order to increase intravascular volume and pressure. In particular, considering treatment directed toward raising the intravascular volume, 9- $\alpha$-fluorohydrocortisone is the first line drug and the only one recommended with the level of evidence ' $C$ ' 


\section{Future Perspectives}

The characterization of the different features of AF in patients with $\alpha$-synucleinopathies remains a priority because this may help to improve the accuracy of the differential diagnoses between these diseases at onset. Since the prognosis for each of these disorders is completely different, an early diagnosis is crucial [34]. Fur- thermore, cardiovascular AF has been demonstrated to have a negative prognostic role in $\alpha$-synucleinopathies [8]; therefore an early detection of cardiovascular dysautonomia will have a positive impact on the disease course by guiding the therapeutic strategy. Finally, an accurate understanding of the pathophysiological basis of AF is needed for the development of new therapies.

\section{References}

1 Skovronsky DM, Lee VM, Trojanowski JQ: Neurodegenerative diseases: new concepts of pathogenesis and their therapeutic implications. Annu Rev Pathol 2006;1:151-170.

2 Mathias CJ: Autonomic diseases: clinical features and laboratory evaluation. J Neurol Neurosurg Psychiatry 2003;74(suppl 3):iii31-iii41.

3 Benarroch E: Overview of the organization of the central autonomic network; in Benarroch E (ed): Central autonomic network: functional organization and clinical correlations, ed 1 Armonk, New York, Futura Publishing Company, Inc., 1997, pp 3-28.

-4 Cersosimo MG, Benarroch EE: Central control of autonomic function and involvement in neurodegenerative disorders. Handb Clin Neurol 2013;117:45-57.

5 Gilman S, Wenning GK, Low PA, et al: Second consensus statement on the diagnosis of multiple system atrophy. Neurology 2008;71: 670-676.

6 Litvan I, Bhatia KP, Burn DJ, et al: Movement Disorders Society Scientific Issues Committee Report: SIC Task Force appraisal of clinical diagnostic criteria for Parkinsonian disorders. Mov Disord 2003;18:467-486.

7 Goldstein DS: Dysautonomia in Parkinson's disease: neurocardiological abnormalities. Lancet Neurol 2003;2:669-676.

8 Fanciulli A, Strano S, Colosimo C, Caltagirone C, Spalletta G, Pontieri FE: The potential prognostic role of cardiovascular autonomic failure in alpha-synucleinopathies. Eur J Neurol 2013;20:231-235.

-9 Freeman R, Wieling W, Axelrod FB, et al: Consensus statement on the definition of orthostatic hypotension, neurally mediated syncope and the postural tachycardia syndrome. Clin Auton Res 2011;21:69-72.

10 Mathias CJ, Mallipeddi R, Bleasdale-Barr K: Symptoms associated with orthostatic hypotension in pure autonomic failure and multiple system atrophy. J Neurol 1999;246:893-898.

11 Wenning GK, Geser F, Krismer F, et al: The natural history of multiple system atrophy: a prospective European cohort study. Lancet Neurol 2013;12:264-274.

12 Calandra-Buonaura G, Guaraldi P, Sambati L, et al: Multiple system atrophy with prolonged survival: is late onset of dysautonomia the clue? Neurol Sci 2013;34:1875-1878.

13 Petrovic IN, Ling H, Asi Y, et al: Multiple system atrophy-parkinsonism with slow progression and prolonged survival: a diagnostic catch. Mov Disord 2012;27:1186-1190.

14 Goldstein DS: Orthostatic hypotension as an early finding in Parkinson's disease. Clin Auton Res 2006; 16:46-54

15 Lipp A, Sandroni P, Ahlskog JE, et al: Prospective differentiation of multiple system atrophy from Parkinson disease, with and without autonomic failure. Arch Neurol 2009;66: 742-750.

16 Velseboer DC, de Haan RJ, Wieling W, Goldstein DS, de Bie RM: Prevalence of orthostatic hypotension in Parkinson's disease: a systematic review and meta-analysis. Parkinsonism Relat Disord 2011;17:724-729.

17 Horimoto Y, Matsumoto M, Akatsu H, et al: Autonomic dysfunctions in dementia with Lewy bodies. J Neurol 2003;250:530-533.

18 Low PA: Composite autonomic scoring scale for laboratory quantification of generalized autonomic failure. Mayo Clin Proc 1993;68: 748-752.

19 Thaisetthawatkul P, Boeve BF, Benarroch EE, et al: Autonomic dysfunction in dementia with Lewy bodies. Neurology 2004;62:1804-1809.

20 Iodice V, Lipp A, Ahlskog JE, et al: Autopsy confirmed multiple system atrophy cases: Mayo experience and role of autonomic function tests. J Neurol Neurosurg Psychiatry 2012;83:453-459.

21 Kaufmann H, Nahm K, Purohit D, Wolfe D: Autonomic failure as the initial presentation of Parkinson disease and dementia with Lewy bodies. Neurology 2004;63:1093-1095.

22 Stuebner E, Vichayanrat E, Low DA, Mathias CJ, Isenmann S, Haensch CA: Twenty-four hour non-invasive ambulatory blood pressure and heart rate monitoring in Parkinson's disease. Front Neurol 2013;4:49.

23 Ziemssen T, Reichmann H: Cardiovascular autonomic dysfunction in Parkinson's disease. J Neurol Sci 2010;289:74-80.

24 Lahrmann H, Cortelli P, Hilz M, Mathias CJ, Struhal W, Tassinari M: EFNS guidelines on the diagnosis and management of orthostat- ic hypotension. Eur J Neurol 2006;13:930936.

25 Schmidt C, Herting B, Prieur S, et al: Valsalva manoeuvre in patients with different Parkinsonian disorders. J Neural Transm 2009;116: 875-880.

26 Oka H, Morita M, Onouchi K, Yoshioka M, Mochio S, Inoue K: Cardiovascular autonomic dysfunction in dementia with Lewy bodies and Parkinson's disease. J Neurol Sci 2007; 254:72-77.

27 Kimpinski K, Iodice V, Burton DD, et al: The role of autonomic testing in the differentiation of Parkinson's disease from multiple system atrophy. J Neurol Sci 2012;317:9296.

28 Holmberg B, Kallio M, Johnels B, Elam M Cardiovascular reflex testing contributes to clinical evaluation and differential diagnosis of Parkinsonian syndromes. Mov Disord 2001;16:217-225.

-29 Asahina M, Young TM, Bleasdale-Barr K, Mathias CJ: Differences in overshoot of blood pressure after head-up tilt in two groups with chronic autonomic failure: pure autonomic failure and multiple system atrophy. J Neurol 2005;252:72-77.

30 Treglia G, Cason E, Stefanelli A, et al: MIBG scintigraphy in differential diagnosis of Parkinsonism: a meta-analysis. Clin Auton Res 2012;22:43-55

-31 Sakakibara R, Tateno F, Kishi M, Tsuyusaki Y, Terada H, Inaoka T: MIBG myocardial scintigraphy in pre-motor Parkinson's disease: a review. Parkinsonism Relat Disord 2014;20: 267-273.

-32 Orimo S, Suzuki M, Inaba A, Mizusawa $\mathrm{H}$ : 123I-MIBG myocardial scintigraphy for differentiating Parkinson's disease from other neurodegenerative parkinsonism: a systematic review and meta-analysis. Parkinsonism Relat Disord 2012;18:494-500.

33 Kaufmann H, Biaggioni I: Autonomic failure in neurodegenerative disorders. Semin Neurol 2003;23:351-363.

34 Maule S, Milazzo V, Maule MM, Di Stefano C, Milan A, Veglio F: Mortality and prognosis in patients with neurogenic orthostatic hypotension. Funct Neurol 2012;27:101-106. 